\title{
Acceptability of multiple micronutrient supplements by pregnant and lactating women in Mali
}

\author{
Víctor M Aguayo ${ }^{1, *}$, Diakalia Koné ${ }^{2}$, Sory lbrahim Bamba ${ }^{3}$, Baba Diallo $^{3}$, \\ Yacouba Sidibé ${ }^{3}$, Diakalia Traoré ${ }^{3}$, Pierre Signé ${ }^{4}$ and Shawn K Baker ${ }^{5}$ \\ 'UNICEF Regional Office for West and Central Africa, BP 29720, Dakar-Yoff, Senegal: ${ }^{2}$ Helen Keller International \\ (HKI), Bamako, Mali: ${ }^{3}$ Ministry of Health, Bamako, Mali: ${ }^{4}$ UNICEF, Bamako, Mali: ${ }^{5} \mathrm{HKI}$, Regional Office for Africa, \\ Dakar, Senegal
}

Submitted 18 November 2003: Accepted 4 August 2004

\begin{abstract}
Background: In Mali, an estimated $73 \%$ of pregnant women are anaemic largely due to iron deficiency. National policy recommends women to take iron and folic acid supplements daily from first prenatal contact until 3 months postpartum. However, many pregnant women in Mali could benefit from multiple micronutrient supplements.

Objective: To assess pregnant women's acceptability of and adherence to a daily multiple micronutrient supplementation scheme compared with the current daily iron and folic acid supplementation scheme.

Design: Seventy pregnant women were allocated to either the daily multiple micronutrient or daily iron and folic acid supplementation scheme. Women started receiving supplements at the end of the first trimester of pregnancy until delivery and throughout the first 3 months postpartum.

Results: No significant differences were observed between comparison groups with respect to women's perceptions about supplement size, colour, taste or flavour. Adherence to the multiple micronutrient supplementation scheme was better (257.5 \pm 20.9 tablets; average adherence 95.4\%) than that to the iron and folic acid supplementation scheme ( $238.5 \pm 32.7$ tablets; average adherence $92.2 \% ; P=0.008$ ) although both were very good, as were women's perceptions about the benefits of micronutrient supplements to their health and that of their newborns.

Conclusion: Malian women adhere to prenatal/postpartum micronutrient supplementation - no matter what supplement is chosen - when access to supplements is guaranteed and when they are provided with minimum, consistent and easily understandable information and counselling, indicating that these are key elements to ensure effective programmes. These findings, together with those of the global research agenda on the efficacy of multiple micronutrient supplements for pregnant women, will inform policy development in Mali for the effective control of iron deficiency and iron-deficiency anaemia in pregnant women.
\end{abstract}

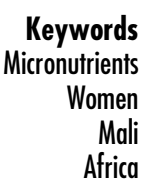

The most recent Demographic and Health Survey in Mali showed that $73 \%$ of pregnant women are anaemic ${ }^{1}$. The high prevalence of anaemia among pregnant Malian women is largely attributable to the poor micronutrient quality of their diets. This because the consumption of ironrich foods (red meat, liver) and iron-fortified foods is limited, and low-cost staple foods such as rice, millet and cassava do not provide enough iron to meet the recommended iron intakes for pregnant women. Moreover, an increasing body of evidence shows that sub-optimal dietary patterns result in low intakes of micronutrients other than iron, leading to the coexistence of multiple micronutrient deficiencies. For example, the recent national survey found that $6.7 \%$ of Malian rural women (in Mali, $75 \%$ of the female population lives in rural areas) experienced night blindness during their most recent pregnancy $^{1}$. This prevalence is higher than the $5 \%$ cut-off value above which maternal vitamin A deficiency is considered a problem of public health significance ${ }^{2}$. Following international guidelines, current national policy in Mali recommends that pregnant women take daily supplements containing iron $(60 \mathrm{mg})$ and folic acid $(400 \mu \mathrm{g})^{3}$. Supplementation should start at the first prenatal contact and end 3 months after delivery. However, such women and their newborns could potentially benefit from a supplement containing multiple vitamins and minerals. 
The Ministry of Health in Mali is therefore considering replacing the current iron and folic acid prenatal supplements with a prenatal supplement that would contain iron, folic acid, vitamins $\mathrm{A}, \mathrm{B}_{1}, \mathrm{~B}_{2}, \mathrm{~B}_{6}, \mathrm{~B}_{12}, \mathrm{C}, \mathrm{D}$ and $\mathrm{E}$, niacin, zinc, copper, selenium and iodine. The formulation of this multiple micronutrient supplement for pregnant women, currently used in several pilot projects world-wide, was agreed upon after taking into consideration the best available information about efficacy, toxicity levels, nutrient costs and potential side-effects related to supplement intake ${ }^{4}$. The objective of the present study was to assess the acceptability of and adherence to the daily multiple micronutrient supplementation scheme versus the current (standard) daily iron and folic acid supplementation scheme by pregnant and lactating Malian women.

\section{Methods}

The study was conducted between June 2001 and June 2002 in Koulikoro and Kolokani, two health districts in the proximity of Bamako, Mali's capital. Seventy pregnant women aged 18-40 years, in their first trimester of pregnancy, gave informed consent to participate in the study. Women who participated in the study in Koulikoro $(n=35)$ took daily the standard iron and folic acid supplement (60 mg elemental iron and $400 \mu \mathrm{g}$ folic acid). Women who participated in the study in Kolokani ( $n=35)$ took daily the multiple micronutrient supplement containing 15 vitamins and minerals. Women did not know whether the prenatal supplement they were taking contained iron and folic acid alone or multiple micronutrients. At the beginning of the second trimester of pregnancy, each woman received a sachet containing 90 tablets (a 3-month supply). The sachet was homedelivered by a health worker from the nearest heath centre, who advised the woman to:

1. take one tablet (and only one) daily for the benefit of her health and that of her baby;

2. take the tablet before going to bed to minimise potential undesirable effects;

3. drink a glass of water to help swallow the tablet; and

4. keep the tablets out of the reach of children.

Each woman received a second and a third sachet (90 tablets each) 3 and 6 months later, respectively. When the second and third supplement sachets were delivered to a pregnant woman, the health worker would:

1. remind the woman of the four (and only the four) recommendations mentioned above;

2. request that the woman return the supplements she had not taken during the previous 3 months; and

3. record the number of supplements returned by the woman.

This information was used to calculate women's reported adherence to the supplementation scheme during pregnancy and in the early postpartum period. At enrolment, semi-structured interviews were conducted to collect information on women's reproductive history, access to health services and knowledge about anaemia. After delivery and at the end of the first 3 months postpartum, semi-structured in-depth interviews were conducted to gather information on perceived benefits and/or undesirable effects of the supplementation scheme. Statistical significance was checked using Student's $t$-test of means for normally distributed variables and chi-square tests of proportions for categorical variables.

\section{Results}

A total of 70 women participated in the study, 35 in each site. All 35 women from Koulikoro (iron and folic acid group) completed the study. In Kolokani (multiple micronutrient group) 30 of the 35 women who enrolled completed the study. The reason for dropping out of the study in all five cases was relocation to a different community (in two cases the woman married and relocated to her husband's community; in three cases the relocation involved all members of the woman's household); these five women were not significantly different from those who completed the study in their group. Results presented in this section concern the 65 women who completed the study.

Women's median age at enrolment was 26 years (range 18-39). Their median number of pregnancies was 4 (range $0-10$ ) and their median number of living children was 3 (range $0-8$ ). The median age of the women's youngest child was 26 months (range 1-72). No significant differences were observed between women in the two comparison groups with respect to these variables. The average walking distance to the nearest health facility was significantly longer for women in the multiple micronutrient group than for women in the iron and folic acid group (109.1 \pm 52.9 vs. $35.3 \pm 36.4 \mathrm{~min} ; \quad P<0.05)$. Similarly, the percentage of women who delivered their youngest living child in a health facility was lower in the multiple micronutrient group than in the iron and folic acid group ( 45 vs. $84 \% ; P<0.05$ ). Women in the two comparison groups did not differ with respect to their knowledge about anaemia: 53 women (82\%) did not know the cause(s) of anaemia. The most frequently reported manifestations of anaemia were dizziness ( 47 women) and weakness (20 women). Twenty women (31\%) were not able to mention any consequence of anaemia for the mother; among those who did, the most frequently reported were prolonged labour (26 women) and weakness (eight women). Nineteen women (29\%) were not able to mention any consequence of anaemia to the newborn; among those who did, the most frequently reported were miscarriage (28 women) and 'weakness' (12 women). 
No significant differences were observed between comparison groups with respect to the women's perceptions about supplement size, colour, taste or flavour (Table 1). Sixty-one women (94\%) found that the size of the supplement was acceptable; 59 women (91\%) found the colour of the supplement acceptable; and 57 women (88\%) found that the taste of the supplement was acceptable; however, 58 women (89\%) found that the supplement 'smelt bad'. Sixty-three of the 65 women (97\%) reported that it was easy for them to remember to take the supplements daily. Thirty-nine of them (60\%) said that they were encouraged to do so by other members of the family or community (in 33 cases by their husbands); $23(35 \%)$ reported that they were neither encouraged nor discouraged to take the supplements; and three (5\%) reported that they were discouraged to do so.

Table 2 shows that the average number of supplements taken during pregnancy (second and third trimesters) was not significantly different between the multiple micronutrient and iron and folic acid groups (168.7 $\pm 21.2 \mathrm{vs}$. $171.8 \pm 11.3$; adherence 93.7 vs. $95.4 \% ; P=0.47)$. On the contrary, the average number of supplements taken during the first 3 months postpartum was significantly higher in the multiple micronutrient group than in the iron and folic acid group (88.8 \pm 4.1 vs. $77.9 \pm 16.4$; adherence 98.6 vs. $86.6 \% ; P=0.001$ ). The average number of supplements taken throughout the 9-month supplementation period was significantly higher in the multiple micronutrient group than in the iron and folic acid group ( $257.5 \pm 20.9$ vs. $238.5 \pm 32.7$; adherence 95.4 vs. $92.2 \%$; $P=0.008)$.

No significant differences were observed between comparison groups with respect to women's perceived benefits of micronutrient supplementation. All 65 women found that supplements had a beneficial effect on their pregnancy. Among the benefits most frequently reported were supplements eliminated some pregnancy side-effects such as headaches, dizziness, nausea and/or vomiting (29 women), feeling healthy (23 women), increased appetite (13 women) and working better (13 women). Sixty-one women (94\%) reported that supplements had a beneficial effect to their delivery. Among the benefits most frequently mentioned were delivery was without complications ( 34 women), delivery was 'quick' (22 women) and delivery was 'easy' (21 women). Sixty-one women (94\%) reported that supplements had a beneficial effect on their newborn. The benefits most frequently mentioned were the newborn/baby is healthy ( 56 women), the baby is big (25 women) and the baby breast-feeds well (nine women). Forty-six women (84\% out of 55 for whom this was not the first pregnancy) found that their newborn was healthier than their previous one; 43 of them (78\%) believed that this was related to the supplements they took during pregnancy. Similarly, 36 women (66\% out of 55 for whom this was not the first pregnancy) found that their newborn was longer than their previous one; 34 of them (62\%) believed that this was due to the supplements they took during pregnancy. No significant differences were observed between comparison groups with respect to women's perceived undesirable effects of micronutrient supplementation. Twenty women (31\%) - eight in the multiple micronutrient group and 12 in the iron and folic acid group - reported that some nausea (14 women) and/or vomiting (12 women) episodes during pregnancy were attributable to the supplement. All 54 women who took supplements during the first 3 months postpartum reported that supplements had beneficial effects on them. The benefits most frequently mentioned were 'I feel healthy' (19 women), 'I feel good' (18 women) or 'I don't fall sick' (18 women). Fifty-two women (96\%) reported that the supplements they took during the first 3 months postpartum had beneficial effects on their infant. The

Table 1 Women's perceptions of supplement size, taste, colour and flavour, by comparison group

\begin{tabular}{lcc}
\hline & $\begin{array}{c}\text { Multiple } \\
\text { micronutrient } \\
\text { group }(n=30)\end{array}$ & $\begin{array}{c}\text { Iron and folic acid } \\
\text { group }(n=35)\end{array}$ \\
\hline -value \\
Supplement was too big & 1 & 0 \\
Supplement was too small & 0 & 3 \\
Supplement's size was adequate (neither too small nor too big) & $29(97 \%)$ & $32(91 \%)$ \\
Supplement's taste was good & 7 & 8 \\
Supplement's taste was bad & 5 & 3 \\
Supplement's taste was neither good nor bad & 18 & 24 \\
Supplement's taste was acceptable & $25(83 \%)$ & $32(91 \%)$ \\
Supplement's colour was nice & 23 & 25 \\
Supplement's colour was ugly & 3 & 3 \\
Supplement's colour was neither nice nor ugly & 4 & 7 \\
Supplement's colour was acceptable & $27(90 \%)$ & $32(91 \%)$ \\
Supplement's smell was good & 0.322 \\
Supplement's smell was bad & 29 & 2 \\
Supplement's smell was neither good nor bad & 1 & 29 \\
Supplement's smell was not acceptable & $29(97 \%)$ & 4 \\
\hline
\end{tabular}


Table 2 Adherence to supplementation scheme, by comparison group

\begin{tabular}{lccc}
\hline & $\begin{array}{c}\text { Multiple } \\
\text { micronutrient } \\
\text { group }(n=30)\end{array}$ & $\begin{array}{c}\text { Iron plus folic acid group } \\
(n=35)\end{array}$ & $P$-value \\
\hline Prenatal supplementation (second and third trimesters of pregnancy) & & \\
Average number of supplements taken & $168.7 \pm 21.2$ & $171.8 \pm 11.3$ & 0.47 \\
Average adherence (\%) & 93.7 & 95.4 \\
Percentage of women with adherence $\geq 90 \%$ & $80(n=24)$ & $86(n=30)$ \\
Postpartum supplementation (first 3 months postpartum) & & \\
Average number of supplements taken & $88.8 \pm 4.1$ & $87.9 \pm 16.4$ \\
Average adherence (\%) & 98.6 & $57(n=20)$ \\
Percentage of women with adherence $\geq 90 \%$ & $93(n=28)$ & $238.5 \pm 32.7$ \\
Nine-month supplementation period & & 92.2 \\
Average number of supplements taken & $257.5 \pm 20.9$ & $60(n=21)$ \\
Average adherence (\%) & 95.4 & 0.001 \\
Percentage of women with adherence $\geq 90 \%$ & $83(n=25)$ & 0.008 \\
\hline
\end{tabular}

benefits most frequently reported were 'The baby is/stays healthy' ( 48 women) and 'The baby breast-feeds well/a lot/frequently' (35 women). No undesirable effects of postpartum micronutrient supplementation were recorded in either group.

\section{Discussion and conclusion}

In Mali, almost three in four pregnant women are anaemic, largely due to iron deficiency. Thus, improving iron intake must be an integral part of a comprehensive strategy to improve the iron status of pregnant Malian women. Unfortunately, economic constraints, poor infrastructures and seasonal harvests limit the access of Malian women to iron-rich foods, especially those of animal origin. Moreover, the lack of widely consumed iron-fortified foods, a key element in the control of iron deficiency in many industrialised and developing countries, justifies further the need for effective iron supplementation programmes, particularly during pregnancy. However, in the light of recent epidemiological evidence showing the coexistence of multiple micronutrient deficiencies in pregnant women ${ }^{1}$ and the anticipated higher biological efficacy of multiple micronutrient supplements ${ }^{5}$, multiple micronutrient supplementation could become a critical component of improved prenatal care in Mali if women's acceptability of and adherence to the multiple micronutrient supplementation scheme is as good as or better than that to the current iron and folic acid scheme.

In our study, adherence to the multiple micronutrient supplementation scheme was better than to iron and folic acid supplementation, although both were very high. Women's acceptability of micronutrient supplements multiple micronutrients or iron and folic acid - was very good, as were women's perceptions about the benefits of micronutrient supplements on their health and that of their newborns. Our study shows that Malian pregnant women adhere to prenatal/postpartum micronutrient supplementation - no matter which supplement is chosen - when access to supplements is guaranteed and they are provided with minimum, consistent, relevant and easily understandable information and counselling. These appear to be key elements to ensure effective prenatal/ postnatal supplementation programmes in Mali and are in accordance with observations made elsewhere ${ }^{6}$.

Our findings, together with those from the global research agenda on the efficacy of multiple micronutrient supplements for pregnant women, will inform policy development in Mali for the effective control of micronutrient deficiencies in pregnant women.

\section{Acknowledgements}

Funding source: This paper is a product of Helen Keller International with support from The United Nations Children's Fund (UNICEF)-Mali and The Micronutrient Initiative (MI), Ottawa, Canada. The opinions expressed in this paper are those of the authors and do not necessarily reflect the views of UNICEF or MI.

\section{References}

1 EDSM-III. Enquête Démographique et de Santé au Mali 2001. Cellule de Planification et de Statistique du Ministère de la Santé (CPS/MS; Bamako) and Direction Nationale de la Statistique et de l'Informatique (DNSI; Bamako) in collaboration with DHS + Program (ORC Macro; MD, USA), 2002.

2 International Vitamin A Consultative Group (IVACG). Maternal Night Blindness: A New Indicator of Vitamin $A$ Deficiency. IVACG Statement 2002. Washington, DC: International Life Sciences Institute Research Foundation, 2002.

3 Stoltzfus R, Dreyfuss M. Guidelines for the Use of Iron Supplements to Prevent and Treat Iron Deficiency Anemia. Washington, DC: International Anemia Consultative Group/ World Health Organization/United Nations Children's Fund, 1998.

4 United Nations Children's Fund (UNICEF)/World Health Organization (WHO)/United Nations University (UNU) 
Composition of a Multi-micronutrient Supplement to be used in Pilot Programs among Pregnant Women in Developing Countries. Report of a UNICEF/WHO/UNU Workshop held at UNICEF Headquarters, New York, 9 July 1999. New York: UNICEF, 1999.

5 Huffman SL, Baker J, Schumann J, Zehner ER. The case for promoting multiple vitamin and mineral supplements for women of reproductive age in developing countries. Food and Nutrition Bulletin 1999; 20(4): 379-94.

6 Galloway R, Dusch E, Elder L, Achadi E, Grajeda R, Hurtado E, et al. Women's perceptions of iron deficiency and anemia prevention and control in eight developing countries. Social Science \& Medicine 2002; 55(4): 529-44 